\title{
Nicotiana glauca whole-genome investigation for cT-DNA study
}

\author{
Galina Khafizova ${ }^{1 *}$, Pavel Dobrynin ${ }^{3,4}$, Dmitrii Polev $^{2}$ and Tatiana Matveeva ${ }^{1}$
}

\begin{abstract}
Objective: Nicotiana glauca (tree tobacco) is a naturally transgenic plant, containing sequences acquired from Agrobacterium rhizogenes by horizontal gene transfer. Besides, N. glauca contains a wide profile of alkaloids of medical interest.

Data description: We report a high-depth sequencing and de novo assembly of $\mathrm{N}$. glauca full genome and analysis of genome elements with bacterial origin. The draft genome assembly is $3.2 \mathrm{~Gb}$, with N50 size of $31.1 \mathrm{kbp}$. Comparative analysis confirmed the presence of single, previously described gT insertion. No evidence was acquired to support idea of multiple T-DNA insertions in the N. glauca genome. Our data is the first comprehensive de novo assembly of tree tobacco and provide valuable information for researches in pharmacological and in phylogenetic fields.
\end{abstract}

Keywords: Nicotiana glauca, Cellular T-DNA, Whole genome sequencing, Genome assembly

\section{Objective}

Nicotiana glauca (tree tobacco) is a member of the Solanaceae family, which includes important crops (potato, tomato, eggplant, pepper) and many medicinal plants [1]. This diploid plant is native to South America and is one of the first Nicotiana species with Agrobacterium cellular T-DNA (cT-DNA) [2]. Its cT-DNA is a partial, inverted repeat, called gT [3]. Tree tobacco belongs to the section Noctiflorae. Sequencing of the genomes of N. tomentosiformis and N. otophora (section Tomentosae) and N. tabacum (section Nicotiana) allowed the detection of previously unknown multiple cT-DNAs [4], raising the question whether there are other T-DNA insertions in the $N$. glauca. NGS data can help answer this question. Besides, N. glauca contains a profile of alkaloids different from N. tabacum [5]. The plant is used for medicinal purposes. Comparative analysis of genomic data of phylogenetically distant tobacco species will provide valuable information on the genetic basis for various traits, especially secondary metabolism. Our data complement the list of species for the comparative genomics of Nicotiana,

\footnotetext{
*Correspondence: galina.khafizova@gmail.com

1 Department of Genetics and Biotechnology, Saint Petersburg State University, Universitetskaya emb. 7/9, Saint Petersburg 199034, Russia Full list of author information is available at the end of the article
}

which opens up new opportunities for pharmacological and phylogenetic studies.

\section{Data description}

One plant isolate was sequenced on Illumina HiSeq machine, yielding in total $210 \mathrm{~Gb}$ of raw sequence data. De novo assembly resulted in 385116 scaffolds, with N50 and L50 of $31.1 \mathrm{kbp}$ and 27293 respectively. Genome size suggested by K-mer analysis is $2 \mathrm{~Gb}$, while the final size of the assembled genome equaled $3.2 \mathrm{~Gb}$. Comparative analyses of $N$. glauca scaffolds against genome assembly of $N$. tabacum TN90 cultivar strain resulted in $3.2 \mathrm{Gbp}$ of aligned sequences median identity of $88 \%$. T-DNA analysis revealed sequences homologous to agrobacterial genes orf13a, orf13, orf14, $\operatorname{rolC}$, rolB and mis. The fragment of T-DNA obtained in the assembly is organized in an imperfect inverted repeat. The similarity of the nucleotide sequences, that we found, and sequence of gT, previously described by Suzuki [3] was 99\%, while its similarity to Agrobacterium T-DNA is $77-89 \%$. Sequences of PCR fragments, amplified from T-DNA/plantDNA junction areas, coincide with known ones (Acs. AB071335, AB071334). 
Table 1 Overview of data files

\begin{tabular}{lllll}
\hline Label & Name of data file & File types & Data repository and identifier & License \\
\hline Supplementary file 1 & Methodology description & .docx file & https://doi.org/10.6084/m9.figshare.5732427.v1 & CC BY \\
Data file 1 & Parameters for the assembly & .txt file & https://doi.org/10.6084/m9.figshare.5645854.v1 & CC BY \\
Data file 2 & T-DNA database & .fa file & https://doi.org/10.6084/m9.figshare.5754120.v1 & CC BY \\
\hline
\end{tabular}

\section{Methodology}

\section{Sample collection}

Leaf tissue of aseptic plants $N$. glauca was used for DNA extraction, with a modified version of Doyle and Doyle protocol [6], yielding $30 \mathrm{ng} / \mu \mathrm{l}$ of high molecular weight DNA.

\section{Library construction}

Purified genomic DNA from $N$. glauca was used to construct both pair-end and mate pair libraries in order to generate a high coverage de novo assembly. A pair-end library with an insert size of 350 bp was constructed using the TruSeq ${ }^{\circledR}$ Nano DNA Library Prep Reference Guide. To improve resolution of repeats during the assembly stage and scaffolding process, one mate pair library with an insert size of $4 \mathrm{kbp}$ was constructed, according to the Nextera ${ }^{\circledR}$ Mate Pair Library Prep Reference Guide.

\section{Read sequencing, quality analysis and filtering}

Pair-end and mate pair libraries were sequenced on four and two lanes using Illumina HiSeq. Quality of raw reads was analyzed with the FastQC [7] program, followed by filtering and trimming raw PE reads with Trimgalore [8]. Mate pair raw reads were processed and splitted with Nextclip [9] and additionally filtered with Trimgalore [8].

\section{Genome assembly}

The genome was assembled with the MaSuRCA-3.2.2 genome assembler [10], [config in data file 1].

\section{Whole genome alignment of Nicotiana glauca and Nicotiana tabacum}

To identify the location of the $N$. glauca cT-DNA insertion relative to the $N$. tabacum genome, we mapped all $N$. glauca scaffolds to N. tabacum scaffolds downloaded from the Sol Genomics Network [11]. To increase accuracy of alignment we masked all known plant repeat classes and their homologs in the $N$. glauca genome. For repeat identification, we used the RepeatMasker software [12] and the latest Repbase Update library from 09.27.2017. For whole genome alignment, we used the Last software [13].

\section{T-DNA analysis}

The Last software [13] was used to carry out the alignment of the database, containing all known T-DNA-like sequences, that were detected as part of cT-DNA [data file 2], to the $N$. glauca genome. To reaffirm T-DNA/ plantDNA junction areas Long PCR was carried out using "LONG PCR enzyme Mix" (Thermo scientific) according to the instructions for the kit (Table 1).

\section{Limitations}

$85 \%$ of the mate pair library proved to be PCR duplicates, which we filtered before assembling. Low coverage of MP reads resulted in low N50 and big number of contigs and scaffolds. A better quality or/and a bigger number of MP libraries should be used in future to improve the assembly.

\section{Abbreviations}

T-DNA: transferred DNA; PE: pair-end; MP: mate pair.

\section{Authors' contributions}

TM developed the overall project design. GK, PD and TM wrote the paper. GK collected the N. glauca sample and extracted DNA from the sample. GK and DP constructed libraries. DP sequenced the genome of N. glauca. PD assembled the $N$. glauca genome and analyzed whole genome alignments. GK, PD and TM performed CT-DNA analysis. All authors read and approved the final manuscript.

\section{Author details}

${ }^{1}$ Department of Genetics and Biotechnology, Saint Petersburg State University, Universitetskaya emb. 7/9, Saint Petersburg 199034, Russia. ${ }^{2}$ Research Park, Saint Petersburg State University, 17 Botanicheskaya St, Peterhof, Saint Petersburg 198504, Russia. ${ }^{3}$ Theodosius Dobzhansky Center for Genome Bioinformatics, Saint Petersburg State University, 41A Sredniy Ave, Saint Petersburg 199004, Russia. ${ }^{4}$ National Zoological Park, Smithsonian Conservation Biology Institute, 3001 Connecticut Ave NW, Washington, DC 20008, USA.

\section{Acknowledgements}

The authors thank Professor Otten (Institut de Biologie Moléculaire des Plantes, Strasbourg) and Professor Lutova (Saint Petersburg University, St. Petersburg) for useful discussion and critical reading of the manuscript.

\section{Competing interests}

The authors declare that they have no competing interests.

\section{Availability of data and materials}

The assembly sequences are available at DDBJ/ENA/GenBank as WGS project under the Accession PGPE00000000. The SRA for whole-genome sequenc ing can be accessed at NCBI SRA via Reference Numbers: SRX3419913, SRX3419914, SRX3419915, SRX3419916, SRX3419917, SRX3419918.

Consent for publication Not applicable. 


\section{Data citation}

1. Khafizova G, Dobrynin P, Polev D. FigShare https://doi.org/10.6084/ m9.figshare.5732427.v1 (2017).

2. Khafizova G, Dobrynin P. FigShare https://doi.org/10.6084/ m9.figshare.5645854.v1 (2017).

3. Khafizova G, Matveeva T. FigShare https://doi.org/10.6084/ m9.figshare.5754120.v1 (2018).

\section{Ethics approval and consent to participate}

Not applicable.

\section{Funding}

This paper was supported by a Grant to Tatiana Matveeva from the Russian Science Foundation 16-16-10010 and a Grant from Saint Petersburg State University №1.52.1647.2016.

\section{Publisher's Note}

Springer Nature remains neutral with regard to jurisdictional claims in published maps and institutional affiliations.

Received: 29 November 2017 Accepted: 5 January 2018

Published online: 12 January 2018

\section{References}

1. Long N, Ren X, Xiang Z, Wan W, Dong Y. Sequencing and characterization of leaf transcriptomes of six diploid Nicotiana species. J Biol Res (Thessalon). 2016;23:6
2. White FF, Garfinkel DJ, Huffman GA, Gordon MP, Nester EW. Sequence homologous to Agrobacterium rhizogenes TDNA in the genomes of uninfected plants. Nature. 1983;301:348.

3. Suzuki K, Ichiro Y, Nobukazu T. Tobacco plants were transformed by Agrobacterium rhizogenes infection during their evolution. Plant J. 2002;32:5.

4. Chen K, Dorlhac de Borne F, Szegedi E, Otten L. Deep sequencing of the ancestral tobacco species Nicotiana tomentosiformis reveals multiple T-DNA inserts and a complex evolutionary history of natural transformation in the genus Nicotiana. Plant J. 2014:80:4

5. Saitoh F, Kawasima N. The alkaloid contents of sixty Nicotiana species. Phytochemistry. 1985;24:477.

6. Doyle JJ, Doyle JL. A rapid DNA isolation procedure for small quantities of fresh leaf tissue. Phytochem Bull. 1987;19:11.

7. FastQC program. https://www.bioinformatics.babraham.ac.uk/projects/ fastqc/. Accessed 12 Jan 2017.

8. Krueger F. Trim Galore!: a wrapper tool around Cutadapt and FastQC to consistently apply quality and adapter trimming to FastQ files. 2015. http://www.bioinformatics.babraham.ac.uk/projects/trim galore/. Accessed 12 Jan 2017.

9. Leggett RM, Clavijo BJ, Clissold L, Clark MD, Caccamo M. NextClip: an analysis and read preparation tool for Nextera Long Mate Pair libraries. Bioinformatics. 2013;30:4

10. Zimin AV, Puiu D, Luo MC, Zhu T, Koren S, Marçais G, Yorke JA, Dvořák J, Salzberg SL. Hybrid assembly of the large and highly repetitive genome of Aegilops tauschii, a progenitor of bread wheat, with the MaSuRCA mega-reads algorithm. Genome Res. 2017;27:5.

11. Sol Genomic Network. https://solgenomics.net. Accessed 25 Feb 2017.

12. Tarailo-Graovac M, Chen N. Using RepeatMasker to identify repetitive elements in genomic sequences. Curr Protoc Bioinform. 2009:25:4-10.

13. Kiełbasa SM, Wan R, Sato K, Horton P, Frith MC. Adaptive seeds tame genomic sequence comparison. Genome Res. 2011;21:3.

\section{Submit your next manuscript to BioMed Central and we will help you at every step:}

- We accept pre-submission inquiries

- Our selector tool helps you to find the most relevant journal

- We provide round the clock customer support

- Convenient online submission

- Thorough peer review

- Inclusion in PubMed and all major indexing services

- Maximum visibility for your research

Submit your manuscript at www.biomedcentral com/submit 